COMMENT. Periorbital bruising is considered a unique complication of dural sinus thrombosis with raised intarcranial pressure. In patient 1 , the bruising had initially been mistaken for a case of assault.

Long-term prognosis of cerebral venous thrombosis (CVST) in childhood was studied in 17 children, aged 1 month to 16 years, at University Medical Center Utrecht, the Netherlands. (De Schryver ELLM, et al. Dev Med Child Neurol August 2004;46:514-519). Mean follow-up was 2 years 8 months. The cause of CVST was mastoiditis in 10, complicated by meningo-encephalitis in 2; 2 had acute lymphatic leukemia, 1 a nonHodgkin's lymphoma, 1 had sickle cell disease, and 1 a malignant histiocytosis and bone marrow transplantation. Twelve presented with raised intracranial pressure, 8 had seizures, and 3 had a focal paresis. The sigmoid sinus was involved in 12, and superior sagittal in 4 . Five died of heart tamponade, meningo-encephalitis, or infection complicating cancer, 3 at an early stage and 2 during follow-up. Mean age of 12 survivors at follow-up was 8 years 7 months (range 2 years 8 months to 20 years). No neurologic deficits occurred in 11, and a mild disability in 1 . Chronic daily headache occurred in 2, and only 1 of 4 survivors with seizures still required AEDs after 1 year follow-up. All of 10 tested had average or above average IQ, 2 had mild learning or attention and behavior problems, and 3 had decreased physical wellbeing. Children who survive CVST have a fair prognosis, and most have normal cognitive and physical development. Mild cognitive deficits and reduction in quality of life are infrequent sequelae.

\title{
CELIAC DISEASE AND STROKE
}

A 3-year-old girl who presented with recurrent episodes of transient hemiplegia and cerebral infarction was diagnosed with asymptomatic celiac disease in a report from Southampton General Hospital, UK. MRI, transcranial Doppler, and MR angiography were abnormal. Tests for celiac disease were positive, despite absence of gastrointestinal symptoms and normal growth and development. Antiendomysial immunoglobulin A antibodies were strongly positive, antitransglutaminase antibodies grossly elevated at over $200 \mathrm{U} / \mathrm{mL}$ (normal 1-15 U/mL), and duodenal biopsy confirmed the diagnosis. After a gluten-free diet, aspirin, and folate supplements, energy level and mood improved (in retrospect the child was considered lethargic and irritable before treatment), and neurodevelopnent was normal at 1-year follow-up. The significant asymmetry between right and left middle cerebral artery velocities on Doppler studies had persisted. (Goodwin FC, Beattie RM, Millar J, Kirkham FJ. Celiac disease and childhood stroke. Pediatr Neurol August 2004;31:139-142). (Respond: Dr Goodwin, Department of Paediatric Neurology, Southampton General Hospital, Tremona Road, Southampton, SO16 6YD, UK).

COMMENT. Stroke has not previously been reported as a complication of celiac disease in childhood. In a recent study of neurologic complications of childhood celiac disease that involved 111 patients (Zelnick et al. Pediatrics June 2004;113:1672-1676; and reviewed in Ped Neur Briefs June 2004;18:46), neurologic disorders were diagnosed in 51\% (cf $20 \%$ controls) and included chronic migraine headache, developmental delay, hypotonia, learning disabilities, ADHD, and epilepsy with occipital calcifications, but not stroke. As with stroke, the occipital lobe seizures may be associated with asymptomatic celiac disease. 